\title{
Ethical challenges in human space missions: a space refuge, scientific value, and human gene editing for space
}

Konrad Szocik, Ziba Norman \& Michael J. Reiss

\begin{abstract}
This article examines some selected ethical issues in human space missions including human missions to Mars, particularly the idea of a space refuge, the scientific value of space exploration, and the possibility of human gene editing for deep-space travel. Each of these issues may be used either to support or to criticize human space missions. We conclude that while these issues are complex and context-dependent, there appear to be no overwhelming obstacles such as cost effectiveness, threats to human life or protection of pristine space objects, to sending humans to space and to colonize space. The article argues for the rationality of the idea of a space refuge and the defensibility of the idea of human enhancement applied to future deep-space astronauts.
\end{abstract}

Keywords: space ethics; space refuge; human mission to Mars; human enhancement; gene editing; bioethics; future studies. 


\section{Introduction}

The idea of human space missions, including missions to Mars, and the establishment of a space colony, including a Mars settlement, are seen as a possible aspect of the human future, especially by the many who are increasingly concerned about the possibility of irreversible damage to the Earth as a planet for human habitation. ${ }^{1}$ While the main challenges of such missions would be technological and medical, there are challenges which deserve the attention of ethicists. The very idea of human presence in space (understood as the long-term persistence of humans beyond the Earth's atmosphere) presents ethical challenges for a number of reasons. One arises from a cost/risk-benefit analysis. While such an analysis is principally concerned with social, political and economic issues, it also entails some ethical questions. In particular, it might be held that there are good reasons to assert that there is no strong rationale for human space missions, including missions to Mars. Such an argument could maintain that there would be substantial and enduring risks without countervailing benefits. Instead of devoting substantial resources (time, money, political effort) to such fanciful journeys, we should urgently face the growing challenges and needs that there currently are on Earth, such as growing overpopulation, potentially irreversible climate change and the threats from nuclear weapons.

In this article, we discuss two possible rejoinders to such objections: the value of a space refuge and the value of scientific exploration of space. Additionally, it is possible that successful human missions to Mars or other distant destinations may require human enhancement. Accordingly, we discuss some issues connected with the idea of substantial/radical biological enhancement, including gene editing of germline and somatic cells. Some kinds of enhancements, such as the use of pharmaceutical countermeasures or

\footnotetext{
${ }^{1}$ Because Mars is the most probable destination for the future human space missions, when we discuss the issue of human space missions, we have in mind mostly missions to Mars. We discuss the significance of Mars, rather than other planets or our Moon, below.

Szocik, K., Norman, Z. \& Reiss, M. J. (2019) Ethical challenges in human space missions: A space refuge, scientific value, and human gene editing for space. Science and Engineering Ethics. DOI: 10.1007/s11948-01900131-1
} 
cyborgization, are likely to be unavoidable if humans are to colonise Mars. While the use of such enhancements raises ethical issues, they are not widely seen as being ethically particularly controversial, so long as they are non-invasive, reversible and non-heritable. However, there are good reasons to treat the idea of radical human enhancements in space as a practical hypothesis, rather than only as one that merits speculative consideration, due to the fact that currently applied countermeasures to hazardous space factors are insufficient for long-term Mars missions. By 'long-term missions' we mean space travel lasting at least three years [this is a shortest estimated duration for the first future human mission to Mars (Szocik et al. 2018)]. It is this that puts missions to Mars and other planets in a different category from missions to the Moon or to space stations that orbit the Earth. It is also worth bearing in mind that effective alternatives for human space missions, such as robotic missions, still present challenges. For fully automated space exploration to operate as effectively as human space exploration requires that space robots have human-like dexterity and intelligence (Szocik and Tachibana in press). However, while present-day robotics cannot match human dexterity, the gap between humans and robots in this respect is narrowing and it is very possible that in a few decades the gap will have closed and that progress in space robotics in the near future will therefore challenge the rationale for human space exploration.

In this article we do not consider in any detail the arguments raised by those environmental ethicists who disagree with human exploration or colonization of space on the grounds that the human species does not have the right to survive, as long as it is the case that humans are not able to live in harmony with the natural world (Smith 2019). Rather, we discuss three issues that can present objections against, as well as arguments for, human missions to Mars and the establishment of a Mars settlement: the idea of a space refuge, the scientific value of space exploration, and the challenge of human enhancement. The aim of this article is to present arguments for human space exploration. We conclude that two of the 
main arguments against sending humans to space - that there is no rationale for a space refuge and that there is a need for a pristine space environment - do not hold under certain conditions. We also discuss the idea of human enhancement for space. This is a new contribution to the space ethics literature, in which human enhancement is considered as an artificial adaptation of humans for survival in space.

\section{The state of the art}

There have been a number of published works in space policy which study the rationale for human space missions. Critics argue that human exploration of space does not offer any benefits for science, industry, medicine or technologies (Szocik 2019; Weinberg 2013). In response, advocates of a human space program focus mostly on benefits for space science (Crawford 2012; Shelhamer 2017) and spinoffs useful for humanity (Crawford 2012; Rovetto 2013, 2016). Some advocates also discuss such non-material benefits provided by human space exploration as human feelings of pride, direct 'access' to the power of universe and so forth (Rovetto 2013, 2016; Shelhamer 2017). One of the strongest arguments for the human presence in space is the concept of a space refuge for post-catastrophic Earth. However, this possibility is only mentioned at the margin of discussions about the existential risks on Earth (Baum 2009; Baum et al. 2015; Jebari 2015; and has recently been critically evaluated (Szocik 2019). The critics argue that a space refuge based in the solar system does not offer advantages which would justify its maintenance. Some of them argue that such a refuge could only play the role of recovery support after a catastrophe on Earth (Baum et al. 2015). This field of research in space policy is open for new arguments to discuss the future place of humans in space, mostly in regard to growing environmental and population challenges on Earth but also technological advances. 
The second relevant field consists of works discussing ethical issues in space. Space ethicists have discussed issues such as whether there is a human right to explore space, environmental ethics, planetary protection, ethics of colonization and other astrobiological matters. Because these issues have been fairly widely discussed (Persson 2012, 2019; Randolph and McKay 2014), in this article we focus on less widely considered ethical issues, such as human enhancement for space (Szocik 2015; Szocik et al. 2019; Szocik and Wójtowicz 2019). The idea of the cyborgization of astronauts is not new (Clynes and Kline 1960). Recently, E. Paul Zehr discussed the concept of human enhancement in the context of space missions, but only at the margin of his study (Zehr 2018). A related issue that we do not discuss in this article, principally because it merits fuller examination than is possible here, is the possible role that advanced AI might play in space colonization, and associated future automatization of space exploration and exploitation (Campa et al. 2019).

\section{The concept of a space refuge}

An argument for space refuge - the concept of Mars as a backup planet - states that humans have a duty to colonize Mars (Munévar 2014; Schwartz 2011), or another space body which is technologically and environmentally feasible, to increase the chances of survival for the human species. This argument finds support in a commonsense logic. We understand intuitively, and every ecologist concerned with the preservation of endangered species would agree, that residing on at least two space bodies is a safer option than on only one. However, because of the challenging nature of such a project, which would require both technological progress and substantial financial investment, the intuitive argument merits rational investigation. Let us for now take it for granted that attempting to ensure the long-term 
survival of the human species is a duty of ours (we return to this claim below). However, this does not imply a particular mechanism. ${ }^{2}$

Ian Stoner discusses the duty to preserve the human species in terms of a dilemma between the colonization of Mars and the protection of Earth, and he argues for the latter alternative (Stoner 2017). If one accepts that humans are obliged to settle space to increase the chances for our species' survival, this goal seems likely to trump other competing values including, for example, the scientific or aesthetic value of a pristine Mars. However, the alternatives of shelters in space and on Earth remain. Advocates of Mars colonization are obliged to find strong reasons to show that space settlement offers extra value when compared with various alternatives of terrestrial shelters, and does not threaten other values such as the risk of contamination of Mars or relinquishing environmental protection of Earth (Stoner 2017).

The simplest response to those who advocate space colonization is that this is an attempt to increase the chances of human survival and should not be seen as an alternative to looking after our Earth. Indeed, it can even be argued that the difficulties - and there will be difficulties - of establishing a durable colony on Mars will increase the efforts that humans make to protecting our own planet as the risks that there is no 'Planet B' become publicly apparent. At the same time, the opposite scenario is possible: humans who learn that a safe life beyond Earth (a Mars settlement) is possible may stop caring about planetary protection on Earth. Opponents of Mars colonization need to show that such a space colony does not offer any added value for protecting humanity. They may argue that even if a successful space

\footnotetext{
${ }^{2}$ It is evident that a space refuge must be a very expensive project. For more arguments on that financial side of the idea of space refuge, and political, social and economic challenges for a public budget policy, see Szocik (2019).

6

Szocik, K., Norman, Z. \& Reiss, M. J. (2019) Ethical challenges in human space missions: A space refuge, scientific value, and human gene editing for space. Science and Engineering Ethics. DOI: 10.1007/s11948-01900131-1
} 
colony is launched somewhere in space, it will not be self-sustainable over a long period of time, and Earth will be still the main homeland for most of humanity. ${ }^{3}$

Reference to terms discussed in existential risk studies may help clear up some conceptual misunderstandings. Alexey Turchin discusses the 'Plan A, Plan B' model for the prevention of catastrophes (Turchin 2018). This model states that plan A is aimed at preventing a catastrophe - a so-called problem-centered approach. Plan B is realized when a catastrophe has already happened and is aimed at providing survival - a so-called consequence-centered approach. Another useful distinction is between improbable and bad plans. While some scenarios are worthy of consideration and logically thought-provoking, they are simply unrealistic - this is the case with improbable plans - or hazardous in the longterm for humanity - they become bad plans. Turchin notes that plan A, both logically and pragmatically, precedes plan B, and this regularity expresses the rule that it is better to prevent than to cope with adverse consequences.

However, Turchin's presumption that prevention is the better and simpler approach to catastrophic risk is not necessarily true for all types of catastrophe. Consider the case of an asteroid impact. It might be supposed that such a scenario can always be prevented - for instance, by asteroids on collision courses with the Earth being destroyed by us before they reach our planet. However, there are good reasons to assume that at some point in the future a collision with an asteroid will be impossible to prevent (e.g. because of errors in the technology to detect and destroy such asteroids or because eventually there is an asteroid too massive to deal with).

Another point arises in regard to the distinction between plan A (prevention) and plan B (treatment), namely that the idea of space colonization is a case which challenges this distinction. While building a space colony requires time and a long-term perspective

\footnotetext{
${ }^{3}$ See the concept of so-called the disposable planet mentality in Schwartz (2019). 
apparently appropriate for a plan A, building a space colony is also an example of plan B's approach, which is aimed at coping with the consequences of an unavoidable catastrophe. Humanity is obligated to apply plan B as soon as it detects an unavoidable anthropogenic or exogenous risk to the survival of humanity on the Earth. In some cases, plan B may mean a duty to leave Earth. In the light of Turchin's distinction between plans A and B, Stoner's dilemma of colonizing Mars or preventing environmental collapse on Earth becomes a false dichotomy. Mars colonization - if considered in terms of a space refuge and not in terms of scientific exploration or resources exploitation - is not a remedy for 'routine' environmental challenges, i.e. environmental challenges other than those which threaten the future of humanity on the Earth.

Turchin identifies three strategies belonging to plan B as 'improving resilience', 'escape' and 'hiding'. Mars colonization falls into the 'escape' strategy. Experts are obligated to identify at least one type of unavoidable exogenous global catastrophe which can be countermanded only by Mars colonization. Two issues arise here. First, the intensity of existential risks and the probability of success of a particular plan come in degrees. For instance, one can estimate that collision with an asteroid of a particular size will cause the death of some proportion of humanity. However, the percentage is not fully predictable for a number of reasons. Survivors of an asteroid impact can die in the months after the collision from such effects as decreasing or increasing temperature, breaks or loss in the food supply, and transmission of illness accelerated by the lack of normally functioning public health services. Therefore, even if one assumes that the immediate consequences of collision with a particular asteroid are not catastrophic for all humanity, focusing only on shelter strategies on Earth may remain too risky an approach. It would be hard to presume that such shelters provide sufficient countermeasures for all possible, mostly long-term, consequences of the impact. 
Secondly, even assuming that the shelter strategy enables a reasonable number of individuals to survive both the initial impact and the various problems in the next few months or even years (such as widespread famines and diseases), there are good reasons to suspect that even if some people are able to survive for a sufficient period of time for climatic conditions to return to near normal (as happens after volcanic winters), it is possible that the long-term quality of their lives and the lives of their descendants will remain very low. A return to the standards of life found in the pre-catastrophic period may be difficult due to technological collapse. It is worth considering the issue of the relative qualities of life in a settlement on Mars and on post-catastrophic Earth. There are good reasons to expect that the quality of life on Mars, at least for the foreseeable future, will be relatively low due to the requirements of living in a space outpost and the very substantial financial costs of providing infrastructure that does more than ensure survival (cf. life on the International Space Station, ISS, since 2000). However, even if the quality of life in a Mars settlement is lower than the quality of life on post-catastrophic Earth, an advantage of a Mars base lies in the fact that Mars settlers will live in relatively stable conditions in contrast to the unpredictable future on post-catastrophic Earth. The idea of a space refuge would work only if such a space settlement would not be threatened by risks and hazards typical for human populations. Some of these hazards have a biological and social genesis and are a part of human social life - such as diseases, social conflicts, psychological problems and challenges of planetary protection. However, at least at the very beginning, when compared with post-catastrophic Earth, living in a space base should be relatively feasible. Of course, a Mars base has an advantage over the Earth only if a Mars outpost is self-sustaining. Any dependence on deliveries from Earth removes the status of a Mars base as a feasible refuge. It is worth keeping in mind that another challenge for Mars as a safe refuge is a risk of an asteroid strike due to its atmosphere, about 100 times thinner than the atmosphere on Earth. 
Some authors, such as Charles Cockell (2002) and Stoner (2017) take for granted that living on Mars will be hard and challenging for daily human life. However, a Mars outpost may be an attractive place to live when compared with life on post-catastrophic Earth (as nuclear bunkers are better than the alternative). Survivors on Earth, previously used to permanently available electricity, food and water and a certain level of security, may, after a catastrophe on Earth, find themselves in conditions where no almost basic needs are satisfied. Their quality of life will change dramatically; aside from physical problems, they may experience stress and traumas as witnesses of ghastly events. In contrast, Mars settlers will live in relatively stable and predictable conditions equipped with a life support system the equivalent of which, back on Earth, depending on the type of catastrophe, will not necessarily be available for survivors. In fact, one of the great advantages of life on Mars is that both those on the base and those who will have spent years getting them there will always have known that conditions will be tough, whereas many of those on Earth will have had no experience, or even anticipation, of life in 'frontier' conditions. However, a large part of the human population lives in remote areas and beyond cities, where the environmental impact of a possible catastrophe may be lower than is the case for city dwellers. Living on a Mars base may not be worse, overall, than life on post-catastrophic Earth but this depends on the type and duration of the catastrophe. ${ }^{4}$ One historical example of catastrophes on Earth were the pandemics of the Black Death in the $14^{\text {th }}$ century which substantially reduced the population of Europe by between a third and a half. Comparable pandemics may be more challenging today due to global migration and, at least theoretically, such pandemics could kill all of humanity.

\footnotetext{
${ }^{4}$ For more detailed discussion on possible catastrophes on Earth and their relation to the idea of space refuge, see Szocik 2019. 
One of the main arguments for a space refuge is the risk of an asteroid impact.

Gonzalo Munévar points out that while an asteroid impact may be thought of as a temporally distant kind of risk, any substantial asteroid impact will cause large-scale damage and the death of up to billions of humans (Munévar 2019). We can add that it is hard to predict the number of victims of an asteroid impact as this depends on its size and on other factors. Potentially, an asteroid impact can kill all of humanity. Even if an asteroid impact does not kill all of humankind, there are good reasons to mitigate its effects by launching one or more space refuges. The main problem with assessing the implications for humanity of a large asteroid impact or any other large-scale space catastrophe lies in the fact that they are only likely to take place in the distant future, possibly hundreds of thousands of years or longer from now (Munévar 2019). Astronomers predict that a collision with an asteroid larger than one kilometer in diameter - large enough to cause global catastrophe - may happen once every few hundred thousand years (Chodas and Yeomans 1999). Whilst it is difficult to influence the political discourse in the case of low probability, high impact events, such as a large asteroid collision with Earth, awareness of possible cosmic threats is growing. In 2016 the United Nations established International Asteroid Day, under resolution [A/RES/71/90], to be observed on June $30^{\text {th }}$ the date of the 1908 Tunguska asteroid impact in Siberia, Russia. However, as Brian Patrick Green points out, the risk of asteroid impact - even if still too distant in time and not too destructive as in the case of Tunguska impact - should not work as a conclusive objection to establishing a space refuge (Green 2019). Human survival requires countermeasures able to protect humans against a number of risks in addition to those posed by space catastrophes, such as those posed by nuclear conflict, climate change or environmental degradation. From a long-term perspective, the arguments in favour of settling humans in space to avoid the risk of extinction have been judged to be strong by Elon Musk (2017), Cameron M. Smith and Evan Davies (2012), David Valentine (2012) and Keith 
Abney (2019). The key idea which lies behind the thinking of such people is that from a very long-term perspective, life on Earth will not be possible. Green argues that an important argument against establishing a space refuge is its possible negative impact on the stability of civilization on Earth (Green 2019). However, as has been argued above, environmental protection on Earth (Plan A) can be realized independently of and simultaneously with investing in a space refuge (Plan B).

Thought-provoking arguments against the idea of a space refuge - at least, arguments which express flexibility and appreciate the context-dependence of this idea - are discussed by Adam Potthast (2019). Potthast argues that while the idea of a space refuge aimed at increasing the chances for survival of humanity is worthy of consideration, there are some situations in which human survival is not an absolute value, so that in some scenarios it would be better for humanity not to survive (cf. Lessing 1982). He refers to a kind of ethics which states that human life should possess some minimal level of quality of life (cf. Derek Parfit's 'repugnant conclusion' (Parfit 1984)). This has been discussed above in regards to a comparison between the quality of life on a Mars base and on post-catastrophic Earth.

In one of his arguments, Potthast makes an analogy with assisted dying. This argument states that there are conditions in which it is better to die than to live. The concept of what we can term an 'inappropriate' quality of life has here at least two meanings. One of them is that humans are morally too bad to have, in all circumstances, the right to life. This meaning applies to arguments discussed by those environmentalists who maintain that humans have only, or mostly, a negative impact on the natural environment and - for this reason - their right to explore new places in space should be limited or forbidden (Billings 2019; Marino 2019; Oman-Reagan 2019). A second meaning of an inappropriate quality of life refers to the conditions of life experienced by humans, as indicated by the analogy with assisted dying. It is hard to compare the quality of life on post-catastrophic Earth and on Mars. The issue is 
further complicated when one attempts to take into account the behavioural challenges of living in a confined and remote environment. Koji Tachibana points out that isolation and confinement are two factors associated with a permanent high risk of stress (Tachibana 2019). They are an integral feature of space outposts. That having been said, individuals vary greatly in the extent to which they find isolation and confinement hard to bear - a fact explored by those responsible for the psychological assessment of putative astronauts (De La $\mathrm{n}$ Torre et al. 2012; Kanas et al. 2009).

In conclusion, the idea of a space refuge is a principal argument for a permanent human presence in space. It can be argued that the great financial cost of such a space refuge is warranted due to the fact that some kinds of possible catastrophes which may occur on Earth may be lethal for all of humanity. At the same time, living in a space refuge will be challenging for a number of reasons. However, living on a post-catastrophic Earth - if this proves possible - may be worse than living on Mars or any other space refuge.

\section{The value of science}

As currently discussed in the literature, the strongest rationale for a human mission to Mars is its expected scientific value. Stoner focuses his attention on the principle of scientific conservation. Following this principle, a researcher does not have a moral right to apply invasive research methods if non-invasive methods are available. If a non-invasive alternative does not exist, only strong moral reasons may justify an invasive approach. Such a strong moral reason is the survival of the human species in a situation in which space resources are necessary to protect humanity. Another (related) criterion is the reversibility / irreversibility of a particular research method. This principle is especially alarming in regards to the risk of contamination of the Mars surface by terrestrial living forms. Stoner concludes that Mars 
colonization will break the principle of scientific conservation due to the fact that any human contact with the Mars surface will lead to contamination of Mars.

However, Stoner's principle of scientific conservation is valid only if one is able to prove that scientific exploration - understood here as an un-crewed exploration aimed at determining if life has ever evolved or currently exists on Mars - as such is an end value. There are good reasons to argue that scientific exploration has only instrumental value which may be protected under special conditions. Let us propose some conditions in which science is given either absolute or secondary priority. Firstly, to be granted absolute priority scientific investigations should be considered valuable for human flourishing. In his thought experiment Joseph Gottlieb considers whether humanity has a right to destroy a precious object, such as a newly-discovered pyramid, if it were demonstrated that in so doing advances would be made leading to a treatment for cancer (Gottlieb 2019). Secondly, scientific exploration should have absolute priority if there are no other socially pressing needs, e.g., environmental protection or the effects of overpopulation. It is worth keeping in mind that the budget for space exploration could potentially be spent directly on problems here on Earth, though this is more a matter for economists than for ethicists. Thirdly, such exploration should be given absolute priority only if humanity does not have any other interests which would necessitate Mars remaining in a pristine state. By this principle, Mars should be protected against any human contamination only if Mars does not offer any other benefits for humans including the value of scientific exploration, the assessment of which as having intrinsic or instrumental value still remains an open question. Included within the concept of human interest, as expressed above, we propose the following: 1) human survival, as expressed by a need for a space refuge; and 2) humanity's right to satisfy a variety of needs, e.g., to explore for the sake of gaining knowledge or obtaining resources. Scientific interests, when compared with human interests, as defined above, should be considered secondary. Obviously, some authors argue that 
scientific interests possess an intrinsic value and have the potential to be beneficial for all of humanity (Schwartz 2020). However, we argue that scientific interest is only an instrumental value, not an end value, and it matters as a secondary human effort. The modified principle of scientific conservation should go as follows: a scientific interest matters only if humanity as such does not find anything interesting or valuable in the exploration of Mars, and if, in fact, Mars has no relevance to non-scientific human interests. If, however, any other competitive non-scientific interest appears, the scientific community is obliged to prove the priority value of scientific interest on Mars if it wishes this scientific interest to trump the non-scientific interest.

Let us assume that there are good moral reasons to colonize Mars. This is a scenario considered by, among others, Gottlieb, who argues that a Mars settlement can offer a reliable refuge and should have priority over the value of scientific exploration - at least under some conditions. However, Gottlieb finds a couple of challenges to the idea of Mars colonization. One of these is the fact that, while many people are aware of the concept of an existential catastrophe, such catastrophes have not occurred in recent years and are not likely in the next few decades. However, they happened in recorded history (see above: pandemics of Black Death). No one is also able to exclude the possibility that some of them, such as massive volcanic eruptions, pandemic diseases, global crop failures, wars of a scale as yet unseen or a string of climate-related disasters, may happen in the foreseeable future. Despite this, it seems that existential risk, as a concept, remains part of futurism but also a part of science-fiction culture, which undermines its weight in today's decision-making. The long-term nature of these existential risks leads to the current situation in which humanity is not mentally prepared for a mission to Mars as a global effort, aimed at building a space refuge. While this mental context and the specificity of a 'social mind' matters, however, it does not undermine our moral duty to colonize space in order to increase the chances for human survival. 
Existential risks provide a good reason for space settlement because human extinction - being irreversible (Turchin 2018) - cuts off all other possibilities of human activity. Human existence is a basic value and the starting point for all other human values and, as such, worthy of protection. In the context of the basic value of human existence, ethical considerations about the possibly higher value of scientific interests and the unique nature of some objects (e.g. the pristine nature of the surface of Mars), as discussed by Stoner and Gottlieb, are inapplicable: such objects have a unique scientific value only as long as the human species exists (e.g. because the pristine Mars is the only (or most likely) place where one can establish whether or not there was (or even is) non-Earth life on Mars). Because only humans are able to detect and assess their value (according to our knowledge, we are the only species able to conduct research and evaluate nature in scientific terms), human survival is a condition sine qua non of any assignment of value to material objects.

It is worth noting that this is an anthropocentric point of view which connects the value of the non-human world to human observer. However, we are far from the position which depreciates any value of the non-human world. Our anthropomorphic approach refers only to particular types of value, namely scientific and ethical. It is hard to presume that nonhuman animals are interested in explanations of the genesis of life on Mars or elsewhere in space, at least in the same way that humans are. While non-human animals appreciate the world as their habitat (for its survival/biological value) and possibly possess some aesthetic biases, they surely do not perceive the world as an object of scientific investigation. Similarly, non-human animals presumably do not have ethical norms or axiological values (but may possess certain moral intuitions, for instance ones to do with what is 'unfair' (de Waal 2018). The world possesses survival and aesthetic value without human observers but we are skeptical as to whether the world possesses scientific and ethical values without humans. 
Another challenge discussed by Gottlieb is possibly more alarming. He points out that maintaining a colony on Mars would be undertaken by the current generation for the benefit of future generations. Current generations may not be able to find an incentive to make any effort for the benefit of people who are not yet born, and who may not be related to them (Parfit 1984) (at least not from the very long-term perspective which would be required for realization of such a multi-generational project as space settlement). Jacob Haqq-Misra rightly calls this effort, if undertaken, a deep-altruism (Haqq-Misra 2019).

The end value of space science is a controversial concept, in that it presupposes the existence of human beings able to undertake science and research and be aware of the value of scientific achievements and discoveries. In a situation of existential risk for humanity, human survival should take priority over the value of a pristine space environment. Space science is of high importance as an instrumental value, however. James S. J. Schwartz points out that it is worth investing in space science as a starting point for future space settlement (Schwartz 2019). Without space science, no space settlement will be possible.

One specific anti-colonization argument is that there is no economic rationale for the exploitation of Martian resources (Stoner 2017). While asteroid mining would be less expensive than a journey to Mars, a mining industry, once established on Mars, may be economically feasible in the long-term. In a longer perspective, space missions may be fully automated, and highly advanced intelligent robots may be able to complete the entire mission without any human intervention (Campa et al. 2019).

\section{The challenge of human enhancement for space travel}

The idea of human enhancement for the purposes of living in space is considered here as an instrumental value. Instrumental value is a value which is assessed more or less in a hierarchy of values dependent on current or future conditions and contexts. As such, it differs from 
absolute value, end value, intrinsic value and/or value per se. End value is always assessed as a high or the highest value, independent of particular conditions. There are good reasons to consider human enhancement for the purposes of living in space - and possibly human enhancements in general - as an instrumental value but not as an end value. Human enhancement for the purposes of living in space may be considered as an instrumental value from the medical and political points of view.

In this section, only one kind of radical human enhancement, gene editing, is considered. Gene editing includes editing of germline and/or somatic cells. The most controversial procedure is gene editing of germline cells (Reiss and Straughan 1996), in that this editing is heritable and thus, for obvious reasons, cannot follow the rule of informed consent; it is also less reversible.

\section{Medical perspective}

Let us assume that current countermeasures applied in space, in mitigation of medical risks, work more or less effectively at the ISS but - when applied to astronauts travelling to or living on Mars - are insufficient. The authors of the recent research on the NASA Twins Study published in Science report two interesting points. First, they point out that "any biological, biophysical, or pharmacological countermeasures that reduce these risks should be considered for development and implementation" (Garrett-Bakelman et al. 2019). While they do not explicitly mention genetic human enhancement and, possibly, were not considering such a scenario, there are good reasons to consider such when evaluating their next observation: the authors state that there are no data on radiation exposure during interplanetary missions longer than one year. However, the hazardous effects of long exposure to space radiation on Mars could be minimized by living underground and/or in appropriate shielded structures. While their statement is, in fact, trivial - because no human astronaut has ever travelled to Mars or indeed spent a couple of months beyond low Earth 
orbit - it shows the limits of current space medicine which does not have knowledge about the full spectrum of risks or the real impact of the deep-space environment on human health. Mission planners are liable to be blamed if they decide to send humans to Mars while not being aware of effective countermeasures. Human enhancement here becomes an obligation rather than an option: such enhancement is akin to a necessary treatment only if humans are sent to Mars. When one applies the therapy/enhancement distinction, human enhancement in space falls into the category of therapy. There are good reasons to consider enhancement in space as a non-trivial procedure aimed at providing a minimal level of wellbeing in a hazardous environment. Human enhancement, including gene editing, becomes, in this case, an instrumental value. The therapy/enhancement distinction is inadequate, however, because some procedures, including reproductive germline gene editing, do not fall clearly into either category (Gyngell et al. 2019).

There are good reasons and promising prospects for the future gene-editing of astronauts. George Church identifies several dozen genes which are responsible for functions and capabilities which may increase human resistance to hazardous space environments. It seems that the most useful genes for humans in space include genes responsible for enhancing bones and bone loss resistance, adaptations for breathing low oxygen atmospheres, decreasing cancer risk, increasing radiation resistance, improving memory and learning, and decreasing levels of stress and anxiety (Church n. d.). Assuming that genes responsible for functions and capacities useful in space will be identified and are open to enhancement, there are strong moral reasons to invest in clinical trials and then to enhance future astronauts. The main targets for gene editing in space include radiation resistance and decreasing the risk of cancer. They can be considered obligatory because of the high risk of cancer and other diseases caused by high exposure to space radiation. The main argument for genetic modification here is the highest value of human life and health which should be protected by all available 
methods. If science and technology make it possible, there are no other equally valid and strong counter-arguments which would be able to prevent human gene editing aimed at increasing and improving human health and protecting human life (when criteria of safety and efficacy are satisfied). Other capacities mentioned above are beneficial but not obligatory, because higher cognitive performance or stress reduction can be achieved by non-invasive methods such as drugs, via pharmacological cognitive enhancement, or may not be essential anyway. However, our current knowledge of genes' functions is still limited. Genetic modifications may cause unpredictable on- and off-target hazardous effects. Progress in genetic sciences is still required to consider seriously (despite many obvious ethical issues) an opportunity to treat somatic and germline gene editing as feasible options, both for therapy and enhancement.

\section{Political perspective}

Paradoxically, the very politicians in spacefaring countries who argue against human enhancement may be its main benefactors. Space missions aim to succeed. The success of human space missions depends on the performance of astronauts. If current risk-mitigation measures are insufficient or there are strong reasons to assume that they are insufficient, mission planners, politicians and the public cannot be sure about mission success. If the weakest part of such a mission is human health and life, rather than the interplanetary spacecraft or life support system, all the stakeholders involved in space missions should be interested in human enhancement in space. Human enhancement thus becomes an instrumental value from a political point of view. It might be objected that the same argument could be made for coal miners, soldiers, workers exposed to radiation or any other occupation where humans are vulnerable. Public policy makers have a right to discuss the idea of human gene editing in regard to such occupations in situations in which other countermeasures do not 
work but people still need to be sent into dangerous environments. However, the issue we are considering in this paper is at a different scale - namely, whether the whole of humanity survives or not. Another substantial difference lies in the fact that while astronauts are exposed all the time to a hazardous space environment, workers on Earth are exposed only temporarily.

\section{Ethical issues in gene editing for the purposes of living in space,}

\section{and other ethical issues in space exploration}

While ethicists and other philosophers interested in radical human enhancement have in the past discussed gene editing only in the abstract, now they have the opportunity to discuss the first (publicized) case of genetically edited humans. In November 2018, the Chinese researcher Jiankui He announced that he edited the embryos of two girls who were born in China (Wang and Yang 2019). His work consisted of editing gene CCR5 which is responsible for enabling HIV to infect human cells. This experiment in gene editing has been broadly condemned by both scientists and philosophers. Scientists argue that there is no medical rationale to justify such a risky intervention. Another criticism lies in the fact that this experiment - based on poor available data - broke the protocols required for medical and genetic procedures (Wang and Yang 2019). The ethical critiques explore mostly one resulting issue: the unpredictable risk for the future development of these baby girls. It is worth mentioning, however, that in the years before this Chinese experiment, philosophers interested in such issues mostly discussed the moral right to conduct gene editing focused on research purposes and disease avoidance (Gyngell 2017; Munsie and Gyngell 2018) rather than germline gene editing for reproductive purposes. Critiques based on unpredictable risk are supported by the fact that - as scholars argue (Wang and Yang 2019) - medical treatments currently in use are sufficient to achieve the results aimed at by gene-editing embryos. 
It is worth considering here two points mentioned above, which may be applied to the discussion of human enhancement for the purposes of living in space. First, human enhancement - in this case, gene editing - must be safe for the patient. Secondly, gene editing should be conducted on future astronauts only when no other solutions are available. It is worth keeping in mind that one of the potential solutions may be a decision to not send astronauts to Mars. Such a decision would be possible when the impact of the space environment is assessed as too hazardous, and no real and reasonable rationale for the expedition would exist. Progress in space robotics may also work as an argument against human gene editing for space. The concept of an instrumental value is relevant here: gene editing should never be treated as an end value or value per se. It should be considered only as necessary under special conditions. These conditions should be as follows: 1) it should be proved that the particular method of gene editing to be used is safe for the patient and the patient's descendants; 2) it should be proved that the intended outcome can only be achieved by gene editing; 3 ) it should be convincingly demonstrated that the intended outcome is such that it is worth undertaking genetic manipulation in order to realise it. These criteria - safety and efficacy - are enumerated by the advocates of a new global moratorium on gene editing of germline cells (Landeret et al. 2019). The distinction is drawn between genetic enhancement and genetic correction - in ethics known better as the therapy/enhancement distinction. In the context of space exploration, as argued above, this distinction is not as clear as it would be on Earth.

Jiankui He broke the rule of informed consent. He did not inform the future parents that the intended gene editing may decrease immunity against many other diseases, such as the West Nile virus, and be harmful for health and life (Cyranoski 2018). The work did not meet criteria of safety and efficacy. The risks, both expected and unexpected, which may be caused by gene editing of germline cells are the main objections raised by Edward Lanphier 
and colleagues (2015). Julian Savulescu notes that He's experiment breaks at least four criteria: safety, necessity, reality and being beneficial (Cyranoski and Ledford 2018). Other scholars argue that gene editing of germline cells should be considered only when no other alternatives are available (Dzau et al. 2019).

Once the scientific community is in possession of safe tools for targeted human gene editing, then ethicists, along with scientists, will be able to examine the moratorium on the application of gene editing: a kind of risk-benefit analysis. Such an examination should take into account the rationale for enhancement, particularly the social and political context, the common good of society, and the time and energy needed to reach a particular target by standard methods when compared to gene editing.

One of the main arguments discussed against human enhancement technologies is the risk of distributive injustice. The argument builds from the premise that the technology will be available (at least in its early stages - like mobile phones) only for the richest, who already possess material advantages over the rest of the population. However - in some sense counterintuitively - human gene editing, like good schooling or a free health service, may reduce the extent of inequality and accompanying injustice. Christopher Gyngell and colleagues note that heritable genome editing can, at least in principle, offer a chance for people who are genetically predisposed to lower intellectual development, by altering this genetic predisposition (Gyngell et al. 2019). While the authors treat this application of gene editing as an example of enhancement, there are good reasons to treat it in terms of genetic correction. The main argument for treating it as an enhancement is that there is no clear minimally-acceptable standard of human intelligence, such that all intelligence levels falling below this standard would be identified as pathological. There is a diversity in human intelligence which is widely acceptable. Under some particular conditions, however, we could argue that there is some minimal required level of human intelligence, such that people 
possessing lower levels of intelligence would be good candidates for genetic correction - after all, we have manipulated children's diets to correct for phenylketonuria for many decades. It can be argued that many of those of lower intelligence than average have distinctive abilities and qualities to contribute to humanity and should be left untreated. Gyngell and colleagues conclude that gene editing of germline cells in some cases is not only morally permissible but even morally required, albeit only after all downside effects are thoroughly understood, since they may be irreversible. The case of enhanced intelligence shows that enhancement procedures may play a therapeutic role, and it is hard to find strong objections against at least some kinds of enhancements.

There are good reasons to assume that human enhancement may be necessary for human interplanetary missions; some such enhancement may be radical and substantial, challenging ethical standards applied in other contexts. There might be a strong argument in favour of radical enhancement if it could reduce the risks of high exposure to space radiation and reduced gravity (Abadie et al. 2015; NASA 2014, 2018; Simonsen and Zeitlin 2017; Zeitlin et al. 2013).

However, it is worth keeping in mind that ethical issues are inevitable in both human and un-crewed mission scenarios. First, the value of human life should be discussed once again. Space ethicists and other space philosophers in collaboration with mission planners should address concerns such as the value of individual life compared with mission success. For instance, should a mission be cancelled - taking into account invested costs, time and energy and putative benefits - when at least one human life is threatened? Other issues include bioethical issues at the beginning and end of life, and the general issue of the ethics of space settlement.

Secondly, new problems will arise when a settlement on Mars becomes a selfsustainable base in which humans reproduce. Ethical concerns will relate to sexual and social 
engineering, a rigorous selection of 'The Right Stuff'" for future Martian offspring, and other restrictions connected with human sexuality in the severe environment. It is worth considering a scenario in which parents of future children born on Mars will have an opportunity - or even an obligation - to edit germline cells of their future offspring following various criteria.

Thirdly, space ethicists and mission planners should consider to what - if any - extent human astronauts may be modified. Do mission planners have a moral responsibility if a deep-space environment is too hazardous for humans, or even a legal responsibility if an appropriate legal code is valid in a particular space-faring country? Should a failure to adopt enhancement procedures, which might have preserved an astronaut's health status, be a legal responsibility of mission planners? The ethical issue arises as to the extent to which mission planners would be responsible for the fact that no enhancements such as gene editing were applied. This topic gets more complicated when one takes into account the social and legal context in which radical enhancements might be prohibited - both in regards to Earth and space as well. While we should not blame mission planners for the non-implementation of human enhancement in situations in which gene editing is prohibited in space-faring countries, it would still be necessary to consider their responsibility for a decision to send humans instead of robots to deep space. We strongly advocate for much needed discussions on these topics. Politics and public policy are intimately connected to ethics, and a mission to Mars offers an opportunity to explore human moral intuitions, flexibility of ethical rules, and their relation to political targets.

We still do not know exactly what kind of medical and psychological effects astronauts will experience in the inhospitable Martian environment of mixed reduced gravity and microgravity, and space radiation. Space scientists have access to medical data from long-

\footnotetext{
${ }^{5}$ T. Wolfe. (1979). The Right Stuff, Farrar, Straus and Giroux. This is a well-researched book involving hundreds of interviews with astronauts and detailing NASA procedures for astronaut selection. Subsequently (1983), it was popularised in the film of the same name.
} 
duration space station missions, including the longest in history, the 437-day space mission of Valeri Polyakov at the Mir space station in 1994/1995, and the one-year ISS mission of Scott Kelly and Mikhail Kornienko in 2015/2016 (Garrett-Bakelman et al. 2019). However, such missions do not offer a full analogy with a human mission to Mars for a number of reasons. First, Polyakov's mission was still substantially shorter than a three-year Mars mission. Secondly, astronauts who return to Earth from the ISS have access to full medical care, in contrast to astronauts who will be landing on Mars. Thirdly, we are not able to predict the impact of a long-term mission to Mars on human health. Because the missions were in low Earth orbit, not beyond, there may be additional radiation impacts associated with a mission to Mars. These and other reasons create a specific ethical and political situation in which mission planners will be not be able fully to assess human physical and psychological health and behavior needs before launching a first human mission to Mars. This is a specific ethical situation of decision making under conditions of uncertainty. There are a couple of ethical rules which should be considered as protocols for space policy and public policy makers. One of them - let us call it the rule of a normative uncertainty - states that if there are some reasons to cancel a particular activity which can harm human health or life, such an activity should be cancelled. Because of uncertainty and expected hazardous factors in space, there are strong reasons at present to postpone human deep-space mission programs [a reasonable alternative seems to be waiting for progress in space robotics (Szocik and Tachibana in press)] or, if they are to take place, to consider the need for radical human enhancement. A decision to send astronauts to Mars in a situation in which they are exposed to high doses of space radiation, and when current countermeasures may be insufficient, may be unethical even if there are strong political reasons to launch a human mission. 


\section{Conclusions}

In this article, we have examined a number of ethical challenges thrown up by the possibility of human space missions: the utility of a space refuge, the value of scientific exploration of space, and the possible need for human enhancement for life in space. The first two have received considerable attention from space philosophers; accordingly, it is worth focusing more on the third issue. There is an increasing tendency to apply technology and medicine for purposes other than medical treatment. While current debates about the ethics of human genetic enhancement are still somewhat theoretical - apart from the recently reported case of the genetically-enhanced Chinese girls - a human mission to Mars offers a real-life space in which human enhancement can be considered as a reasonable option. The concept of human enhancement in space goes beyond the therapy-enhancement distinction customarily discussed in ethics today. While substantial genetic enhancement carried out on Earth for the purposes of those who will live their lives on Earth seems to be more of a choice than a medical necessity - though some ethicists such as Julian Savulescu argue that gene editing aimed at removing diseases from the population is a moral duty - there are good reasons to believe that the substantial enhancement of future Mars astronauts might be necessary to increase their chances of survival. In addition, human enhancement becomes, here, an instrumental value under specific conditions, from a political point of view - that is, if one assumes that mission success requires high-performing astronauts, and that their performance requires enhancement. While there are good reasons - mostly medical and political - to consider human enhancement in space, and enhancement in general, as an instrumental value, there are still good reasons to argue against treating human enhancement as an end value. 


\section{References}

Abadie, L. J., Lloyd, C. W., \& Shelhamer, M. J. (2015). Gravity, Who Needs It? NASA Studies Your Body in Space. Retrieved from: https://www.nasa.gov/content/gravitywho-needs-it.

Abney, K. (2019). Ethics of colonization: arguments from existential risk. Futures, 110, 6063.

Baum, S. D. (2009). Cost-benefit analysis of space exploration: Some ethical considerations. Space Policy, 25, 75-80.

Baum, S. D., Denkenberger, D. C., \& Haqq-Misra, J. (2015). Isolated refuges for surviving global catastrophes. Futures, 72, 45-56.

Billings, L. (2019). Colonizing other planets is a bad idea. Futures, 110, 44-46.

Campa, R., Szocik, K., \& Braddock, M. (2019). Why space colonization will be fully automated. Technological Forecasting and Social Change, 143, 162-171.

Chodas, P. W., \& Yeomans, D. K. (1999). Predicting close approaches and estimating impact probabilities for near-Earth objects. Advances in the Astronautical Sciences, 103.

Church, G. (n. d.). Multigenic traits can have single genes with large impacts. Retrieved from: http://arep.med.harvard.edu/gmc/protect.html.

Clynes, M. E., \& Kline, N. S. (1960). Cyborgs and space. Astronautics, 26-27, 74-76,

Cockell, C. (2002). Mars is an awful place to live. Interdisciplinary Science Reviews, 27(1), 32-38.

Crawford, I. A. (2012). Dispelling the myth of robotic efficiency: why human space exploration will tell us more about the Solar System than will robotic exploration alone. Astronomy and Geophysics, 53, 2.22-2.26.

Cyranoski, D. (2018). Baby gene edits could affect a range of traits. Nature, doi: 10.1038/d41586-018-07713-2.

Cyranoski, D., \& Ledford, H. (2018). Genome-edited baby claim provokes international outcry. Nature, 563, 607-608.

De La n Torre, D. D., van Baarsen, B., Ferlazzo, F., Kanas, N., Weiss, K., Schneider, S., Whiteley, I. (2012). Future perspectives on space psychology: recommendations on psychosocial and neurobehavioural aspects of human spaceflight. Acta Astronautica, $81,587-599$.

De Waal, F. (2019). Prosocial primates: cooperation and empathy, Diversity in HarmonyInsights from Psychology: Proceedings of the 31st International Congress of Psychology, John Wiley \& Sons, 64-84.

Dzau, V. J., et al. (2019). Academies' action plan for germline editing. Nature, 567, 175, doi: 10.1038/d41586-019-00813-7.

Garrett-Bakelman, F. E., et al. (2019). The NASA Twins Study: A multidimensional analysis of a year-long human spaceflight. Science, 364(eaau8650), 127-144.

Gottlieb, J. (2019). Space colonization and existential risk. Journal of the American Philosophical Association (forthcoming).

Green, B. P. (2019). Self-preservation should be humankind's first ethical priority and therefore rapid space settlement is necessary. Futures, 110, 35-37.

Gyngell, C. (2017). Gene editing and the health of future generations. Journal of the Royal Society of Medicine, 110(7), 276-279.

Gyngell, C., Bowman-Smart, H. \& Savulescu, J. (2019). Moral reasons to edit the human genome: Picking up from the Nuffield report. Journal of Medical Ethics, doi:10.1136/medethics-2018-105084. 
Haqq-Misra, J. (2019). Can deep altruism sustain space settlement? In K. Szocik (ed.), The Human Factor in a Mission to Mars, Space and Society. Springer, Cham.

Jebari, K. (2015). Existential risks: exploring a robust risk reduction strategy. Science and Engineering Ethics, 21(3), 541-554.

Kanas, N., G. Sandal, J.E. Boyd, V.I. Gushin, D. Manzey, R. North, G.R. Leon, P. Suedfeld, S. Bishop, E.R. Fiedler, N. Inoue, B. Johannes, D.J. Kealey, N. Kraft, I. Matsuzaki, D. Musson, L.A. Palinkas, V.P. Salnitskiy, W. Sipes, J. Stuster, J. Wang, Psychology and culture during long-duration space missions. Acta Astronautica, 64(7-8), 659-677.

Landeret, E. S., et al. (2019). Adopt a moratorium on heritable genome editing. Nature, 567, 165-168.

Lanphier, E., et al. (2015). Don't edit the human germ line. Nature, 519, 410-411.

Lessing, D. (1982). The Making of the Representative for Planet 8, New York: Alfred A. Knopf.

Marino, L. (2019). Humanity is not prepared to colonize Mars. Futures, 110, 15-18.

Munévar, G. (2014). Space exploration and human survival. Space Policy, 30(4), 197-201.

Munévar, G. (2019). An obligation to colonize outer space. Futures, 110, 38-40.

Munsie, M., \& Gyngell, C. (2018). Ethical issues in genetic modification and why application matters. Current Opinion in Genetics \& Development, 52, 7-12.

Musk, E. (2017). Making humans a multi-planetary species. New Space, 5(2), 46-61.

NASA. (2014). NASA's Journey to Mars. Retrieved from NASA Website: https://www.nasa.gov/content/nasas-journey-to-mars.

NASA. (2018). Space Radiation Risks. Retrieved from NASA Website: https://www.nasa.gov/hrp/elements/radiation/risks.

Oman-Reagan, M. P. (2019). Politics of planetary reproduction and the children of other worlds. Futures, 110, 19-23.

Parfit, D. (1984). Reasons and Persons, Oxford: Clarendon Press.

Persson, E. (2012). The moral standing of extraterrestrial life. Astrobiology, 12, 976-984.

Persson, E. (2019). Ethics for an uninhabited planet, In K. Szocik (ed.), The Human Factor in a Mission to Mars, Space and Society, Springer, Cham.

Potthast, A. (2019). Alien attacks, hell gerbils, and assisted dying: Arguments against saving mere humanity. Futures, 110, 41-43.

Randolph, R. O., \& McKay, C. P. (2014). Protecting and expanding the richness and diversity of life, an ethic for astrobiology research and space exploration. International Journal of Astrobiology, 13, 28-34.

Reiss, M. J., \& Straughan, R. (1996). Improving Nature? The Science and Ethics of Genetic Engineering, Cambridge University Press, Cambridge.

Rovetto, R. J. (2013). The essential role of human spaceflight. Space Policy, 29(4), 225-228.

Rovetto, R. J. (2016). Defending spaceflight - The echoes of Apollo. Space Policy, 38, 6878.

Schwartz, J. S. J. (2011). Our moral obligation to support space exploration. Environmental Ethics, 33(1), 67-88.

Schwartz, J. S. J. (2019). Space settlement: what's the rush? Futures, 110, 56-59.

Schwartz, J. S. J. (2020). The Value of Science in Space Exploration, Oxford: Oxford University Press.

Shelhamer, M. (2017). Why send humans into space? Science and non-science motivations for human space flight. Space Policy, 42, 37-40. 
Simonsen, L. C., \& Zeitlin, C. (2017). Briefing to NAC HEO/SMD Joint Committee Meeting Mars Radiation Environment - what have we learned? July 25 2017, Retrieved from: https://www.nasa.gov/sites/default/files/atoms/files/mars_radiation_environment_nac july_2017_finaltagged.pdf.

Smith, C. M., \& Davies, E. (2012). Emigrating Beyond Earth: Human Adaptation and Space Colonization, New York: Springer.

Smith, K. C. (2019). Homo reductio: eco-nihilism and human colonization of other Worlds. Futures, 110, 31-34.

Stoner, I. (2017). Humans should not colonize Mars. Journal of the American Philosophical Association, 3(3), 334-353.

Szocik, K. (2015). Mars, human nature and the evolution of the psyche. Journal of the British Interplanetary Society, 68(12), 403-405.

Szocik, K. (2019). Should and could humans go to Mars? Yes, but not now and not in the near future. Futures, 105, 54-66.

Szocik, K., \& Tachibana, K. (in press). Ethical considerations of human enhancement and artificial intelligence for space missions. Astropolitics.

Szocik, K., \& Wójtowicz, T. (2019). Human enhancement in space missions: from moral controversy to technological duty. Technology in Society, 59.

Szocik, K., Abood, S., \& Shelhamer, M. (2018). Psychological and biological challenges of the Mars Mission viewed through the construct of the evolution of fundamental human needs. Acta Astronautica, 152, 793-799.

Szocik, K., Campa, R. Rappaport, M. B., \& Corbally, C. (2019). Changing the paradigm on human enhancements. The special case of modifications to counter bone loss for manned Mars Missions. Space Policy, 48, 68-75.

Tachibana, K. (2019). A Hobbesian qualm with space settlement. Futures, 110, 28-30.

Turchin, A. (2018). Approaches to the prevention of global catastrophic risks. Human Prospect, 7(2), 53-65.

Valentine, D. (2012). Exit strategy: profit, cosmology, and the future of humans in space. Anthropological Quarterly, 85(4), 1045-1067.

Wang, H., \& Yang, H. (2019). Gene-edited babies: what went wrong and what could go wrong. PLoS Biol, 17(4), e3000224, doi:10.1371/journal.pbio.3000224.

Weinberg, S. (2013). Response: against manned space flight programs. Space Policy, 29, 229-230.

Zehr, P. E. (2018). Chasing Captain America: How Advances in Science, Engineering, and Biotechnology Will Produce a Superhuman, ECW Press.

Zeitlin, C., et al. (2013). Measurements of energetic particle radiation in transit to Mars on the Mars Science Laboratory. Science, 340(6136), 1080-4.

Szocik, K., Norman, Z. \& Reiss, M. J. (2019) Ethical challenges in human space missions: A space refuge, scientific value, and human gene editing for space. Science and Engineering Ethics. DOI: 10.1007/s11948-01900131-1 